\title{
Recent developments in higher order precision calculations at lower energies
}

\author{
Andrzej Czarnecki* \\ Department of Physics \\ 4-181 CCIS \\ University of Alberta \\ Edmonton AB T6G 2E1, Canada \\ E-mail: andrzejceualberta.ca
}

Opportunities and challenges in bound state calculations are briefly reviewed. As examples, the Lamb shift and the bound-electron gyromagnetic factors are discussed in some detail. Modern algorithms for determining Feynman diagrams will be more easily applied to bound state calculations if we can develop a systematic method of expansions in the external field.

“Loops and Legs in Quantum Field Theory” 11th DESY Workshop on Elementary Particle Physics April 15-20, 2012

Wernigerode, Germany

\footnotetext{
* Speaker.
} 


\section{Introduction}

The competitive advantage of low-energy experiments over those at colliders is the high precision of measurements. New phenomena can be discovered only if predictions are available for the Standard Model contributions to the processes under study. Making those predictions is the task of theorists.

Contemporary experiments in low energy physics often require theoretical predictions for such quantities as the Lamb shift in hydrogen and in muonic atoms, hyperfine splitting in positronium, and loop effects in few-body systems. All these topics refer to some bound states, including exotic atoms and ions. Exotic systems may involve muons instead of electrons, or positrons instead of hadronic nuclei.

Such systems are of considerable current interest. Muonic hydrogen has recently been used to determine the charge radius of the proton [1] and weak formfactors of the nucleon [2, 3]. Muon bound with an aluminum nucleus is a likely system to be used in the near future to search for the muon-electron conversion [4]. Hydrogen-like ions are being used to best determine the electron mass (see [5] and references therein). Another recent success story involving exotic atoms is the determination of the antiproton mass using an exotic variant of helium [6,7].

Static atomic properties that are of particular interests are illustrated with Fig. 1. The average energy level of a spin multiplet is known as the Lamb shift. Spin-spin interaction of an electron and the nucleus gives rise to the hyperfine splitting. And the electron interaction with the nucleus modifies the electron's magnetic moment, parameterized by the gyromagnetic factor $g \simeq 2+$ corrections. It is interesting that these three very different types of effects can be theoretically determined using very similar methods of computing loop calculations, which is one reason why they are of interest to the audience at Loops and Legs.

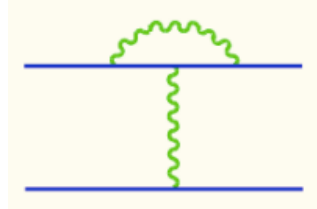

(a)

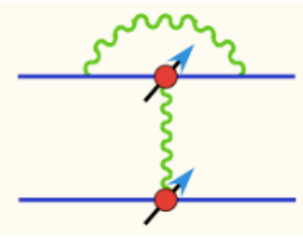

(b)

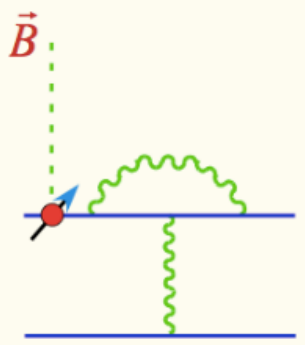

(c)

Figure 1: Static atomic properties of interest in high-precision studies: (a) Lamb shift; (b) hyperfine splitting; and (c) the bound-electron gyromagnetic factor interacting with an external magnetic field. The upper horizontal solid line denotes an electron or a muon, the lower one is the nucleus. Arrows symbolize spin interactions. Wavy lines denote photons.

Binding of an unstable particle such as a muon also modifies its decay properties. This is important for studies of the nuclear muon capture and of the backgrounds to muon-electron conversion searches, where accurate predictions for the bound muon lifetime and the spectrum of the daughter electron are needed.

Studies of static and dynamic properties of bound particles are complicated by the particle motion and by its interaction with the nucleus. Fortunately, some expansion parameters are available 
in the case of the electromagnetic binding to a small or a medium-size nucleus. Binding effects can then be described by a series of powers and logarithms of $Z \alpha$, where $Z$ is the atomic number and $\alpha \simeq 1 / 137$ is the fine structure constant. The nucleus can be treated as a static source of the electric field in the first approximation. Corrections due to the nuclear motion are included as perturbations in an expansion in $m / M$, where $m$ is the mass of the lighter particle such as an electron or a muon, and $M$ is the mass of the nucleus. Finally, effects of electromagnetic self-interactions are parameterized by powers of $\alpha$, just like for a free particle.

Here I use examples of the Lamb shift and of the bound-electron $g$ factor to illustrate recent progress and opportunities for making further advances in bound state calculations.

\section{Lamb shift and the hyperfine splitting}

The gross spectrum of a hydrogen-like atom is described by

$$
E_{n}=\frac{\mu(Z \alpha)^{2}}{2 n^{2}}
$$

where $n=1,2, \ldots$ labels the energy level, $\mu$ is the reduced mass, and $Z$ is the atomic number of the nucleus. This formula receives corrections from relativistic and spin effects, from quantum loops, and from the extended charge distribution in the nucleus. By convention, the effects related to the spin-spin interaction between the electron and the nucleus are called the hyperfine splitting (HFS). In atoms with a hadronic nucleus the magnetic moment of the nucleus is much smaller than that of the electron and the HFS splits an energy level into very close sublevels dependent on the spin state. The average energy of that multiplet of states differs from (2.1) by the Lamb shift.

The lowest order radiative corrections inducing the Lamb shift are shown in Figure 2. Both diagrams represent changes of the Coulomb interaction when the electron is close to the nucleus, thus affecting mainly the $\mathrm{S}$ states.
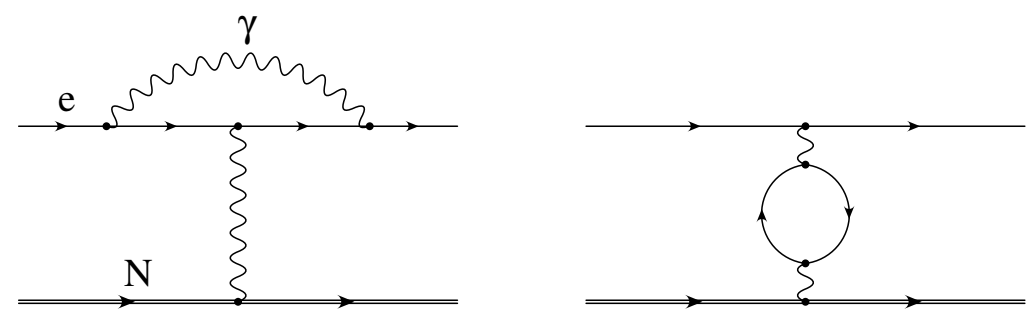

Figure 2: One loop diagram giving rise to Lamb shift.

The vertex correction would be infrared divergent if the electron were free. For the bound electron, the size of the atom cuts off the loop integration; the result contains a positive logarithm of the ratio of the Compton wavelength of the electron to the Bohr radius, $\sim \ln Z \alpha$. This correction, first determined by Bethe [8], weakens the short-distance electrostatic attraction, decreasing the binding energy of the $\mathrm{S}$ states.

The vacuum polarization process has the opposite effect, enhancing the short-distance attraction. It was first evaluated by Uehling [9]. However, for a normal atom there is no logarithmic enhancement and this effect is small. For this reason, in hydrogen-like atoms the negative vertex 
correction dominates and the affected S state lies higher than the P state that corresponds to it (has the same total angular momentum).

In muonic hydrogen, on the other hand, the vacuum polarization has a positive logarithm $\ln \left(m_{\mu} / m_{e}\right)$ and becomes even more important than the vertex correction. For this reason, in the $\mu p$ atom, the $\mathrm{S}$ state lies below the $\mathrm{P}$ state.

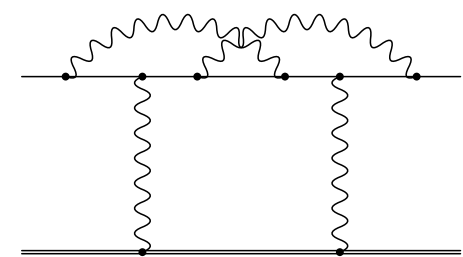

(a)

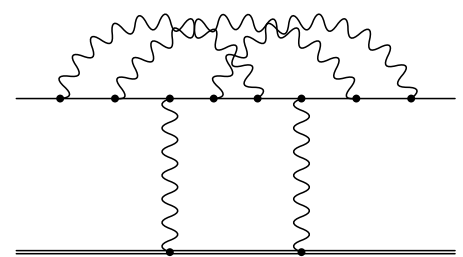

(b)

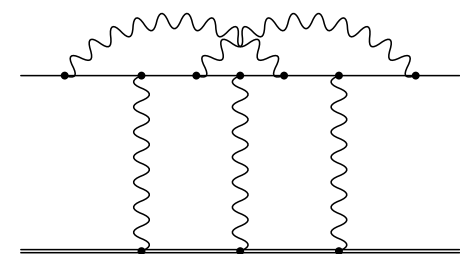

(c)

Figure 3: Examples of multiloop contributions to the Lamb shift.

Since the pioneering work by Bethe, precision of the theoretical prediction of the Lamb shift has greatly improved. Three-loop corrections such as those shown in Fig. 3(a) were first determined in [10 - 12]. Recently, the precision of that result was improved by about two orders of magnitude using modern methods of computing Feynman diagrams [13]. Three-loop corrections include also other types of diagrams; a summary of all contributions, including an analytical result for the vertex correction, can be found in [14].

Current efforts focus on four-loop diagrams, such as those shown in Fig. 3(b,c). The first type, with three self-energy loops and two photons exchanged between the electron and the nucleus, both for the Lamb shift and for the HFS, are being gradually evaluated in a series of papers by Eides and Shelyuto (see [15] for a recent discussion). The second type, with an exchange of three photons with the nucleus, seems to be even more challenging; it is a worthy goal for the modern approaches.

It is interesting that there exists already a numerical evaluation of diagrams with two loop selfenergies and an arbitrary number of exchanges with the nucleus [16, 17]. Unfortunately this approach works well only for relatively large values of $Z$ and it is difficult to use it to determine the correction in hydrogen. Ultimately, it is important that perturbative results for low $Z$ be matched with those numerical determinations.

\section{Bound electron $g$ factor}

Once a method is developed for evaluating contributions to the Lamb shift or the HFS, it can be applied to determine corrections to the gyromagnetic $(g)$ factor of a bound electron. Interesting in its own right, the modification of the $g$ by the binding effects has practical applications to the measurements of the electron mass.

Such measurements are most precise with electrons trapped in a Penning trap [18]. However, the trap being in a finite temperature, the electron interacts with the blackbody radiation and is moving. We know from special relativity that such thermal motion results in a modification of the electron's apparent mass. This tiny effect is in fact an important source of error. The best current measurements of the electron mass $[19,20,5]$ use hydrogen-like ions instead of a free 
electron. This increases the mass of the system by about four orders of magnitude and, at the given temperature, correspondingly decreases the relativistic effects, removing this source of the error.

The energy levels of the ion in a Penning trap depend on its interaction with the electromagnetic fields. Thus, the interpretation of these measurements requires an accurate value of the $g$ factor of the bound electron. In the lowest order, that is neglecting self-interactions of the electron, the effect of the Coulomb field on the $g$ factor was first determined by Breit in 1928 [21], right after the discovery of the Dirac equation (and its explanation why $g \simeq 2$ ). Breit found

$$
g_{\text {bound }}=\frac{2}{3}\left(1+2 \sqrt{1-(Z \alpha)^{2}}\right) \simeq 2-\frac{2}{3}(Z \alpha)^{2}
$$

an effect of the opposite sign to the loop correction found by Schwinger two decades later [22],

$$
\Delta g_{\text {loop }}=\frac{\alpha}{\pi} \text {. }
$$

These two classic results show how the binding effects are described by the parameter $Z \alpha$, while the self-interaction corrections have just powers of $\alpha$.

The progress since those pioneering studies has been slower than for the Lamb shift and the HFS. Only in 2004 were the one loop self-energy corrections to the one-photon exchange with the nucleus evaluated [23]. Two-loop corrections were determined soon afterwards [24]. Corrections to the two-photon exchange are still not fully known, more than half a century after the corresponding precision was reached for the Lamb shift and the HFS [25-27]. Again, there are many opportunities here for applying new developments in the Feynman diagram technology.

\section{Summary}

The last 25 years have seen a spectacular development of computational methods for the Feynman diagrams with free particles. On the other hand, problems involving bound states remain the domain of as much art as science. Not even a good diagrammatic representation of the perturbation theory has been discovered: the same diagram can contribute to various orders in $Z \alpha$ since propagators of charged particles depend on the electromagnetic field.

The first step towards a systematization of bound state calculations should be such a generalization of asymptotic expansions that the field can be treated as an expansion parameter. This is challenging since it does not in general commute with the momentum of the charged particle. Once this difficulty has been overcome, modern algorithms for computing Feynman diagrams will help achieve new levels of precision also for bound state problems.

\section{Acknowledgments}

I thank J. Aslam, D. Bryman, M. Dowling, X. Garcia i Tormo, S. Karshenboim, K. Melnikov, K. Pachucki, and M. Puchalski for collaboration on topics related to this talk. This research was supported by the Science and Engineering Research Canada (NSERC).

\section{References}

[1] R. Pohl, A. Antognini, F. Nez, F. D. Amaro, F. Biraben, et al., The size of the proton, Nature 466 (2010) 213-216. 
[2] MuCap Collaboration Collaboration, V. Andreev et al., Measurement of the rate of muon capture in hydrogen gas and determination of the proton's pseudoscalar coupling $g_{P}$, Phys.Rev.Lett. 99 (2007) 032002, [0704.2072].

[3] MuCap Collaboration Collaboration, V. Andreev et al., Measurement of Muon Capture on the Proton to 1\% Precision and Determination of the Pseudoscalar Coupling $g_{P}$, [1210.6545].

[4] Mu2e Collaboration Collaboration, D. Brown, Mu2e, a coherent $\mu \rightarrow e$ conversion experiment at Fermilab, AIP Conf.Proc. 1441 (2012) 596-598.

[5] S. Sturm, A. Wagner, B. Schabinger, J. Zatorski, Z. Harman, W. Quint, G. Werth, C. H. Keitel, and K. Blaum, g factor of hydrogenlike ${ }^{28} \mathrm{Si}^{13+}$, Phys. Rev. Lett. 107 (Jul, 2011) 023002.

[6] ASACUSA Collaboration Collaboration, M. Hori et al., Two-photon laser spectroscopy of antiprotonic helium and the antiproton-to-electron mass ratio, Nature 475 (2011) 484-488.

[7] R. S. Hayano, M. Hori, D. Horvath, and E. Widmann, Antiprotonic helium and CPT invariance, Rep. Prog. Phys. 70 (2007) 1995.

[8] H. A. Bethe, The electromagnetic shift of energy levels, Phys. Rev. 72 (1947) 339-341.

[9] E. A. Uehling, Polarization effects in the positron theory, Phys. Rev. 48 (1935) 55-63.

[10] K. Pachucki, Complete two-loop binding correction to the Lamb shift, Phys. Rev. Lett. 72 (1994) 3154.

[11] M. I. Eides and V. A. Shelyuto, New corrections to hyperfine splitting and Lamb shift and the value of the Rydberg constant, Pisma Zh.Eksp.Teor.Fiz. 61 (1995) 465-470. [JETP Lett. 61, 478 (1995)]].

[12] M. I. Eides and V. A. Shelyuto, Corrections of order $\alpha^{2}(Z \alpha)^{5}$ to hyperfine splitting and Lamb shift, Phys. Rev. A52 (1995) 954, [hep-ph/9501303].

[13] M. Dowling, J. Mondéjar, J. H. Piclum, and A. Czarnecki, Radiative-nonrecoil corrections of order $\alpha^{2}(Z \alpha)^{5}$ to the Lamb shift, Phys. Rev. A81 (2010) 022509, [0911. 4078].

[14] K. Melnikov and T. van Ritbergen, The three loop slope of the Dirac form factor and the 1S Lamb shift in hydrogen, Phys. Rev. Lett. 84 (2000) 1673-1676, [hep-ph/9911277].

[15] M. I. Eides and V. A. Shelyuto, Three-Loop Contribution to Hyperfine Splitting in Muonium: Polarization Corrections to Light by Light Scattering Blob, Phys.Rev. A86 (2012) 024501, [1207.1021].

[16] V. Yerokhin, P. Indelicato, and V. Shabaev, Two loop selfenergy correction in high Z hydrogen - like ions, Phys.Rev.Lett. 91 (2003) 073001, [hep-ph/ 0302268$].$

[17] V. A. Yerokhin, P. Indelicato, and V. M. Shabaev, Two-loop self-energy contribution to the Lamb shift in H-like ions, Phys. Rev. A71 (2005) 040101, [hep-ph / 0411348 ].

[18] D. L. Farnham, R. S. Van Dyck, Jr., and P. B. Schwinberg, Determination of the electron's atomic mass and the proton/electron mass ratio via Penning trap mass spectroscopy, Phys. Rev. Lett. 75 (1995) 3598.

[19] H. Häffner, T. Beier, N. Hermanspahn, H. Kluge, W. Quint, S. Stahl, J. Verdú, and G. Werth, High-accuracy measurement of the magnetic moment anomaly of the electron bound in hydrogenlike carbon, Phys. Rev. Lett. 85 (2000) 5308.

[20] J. Verdú, S. Djekic, S. Stahl, T. Valenzuela, M. Vogel, G. Werth, T. Beier, H. Kluge, and W. Quint, Electronic g factor of hydrogenlike oxygen ${ }^{16} \mathrm{O}^{7+}$, Phys. Rev. Lett. 92 (2004) 093002.

[21] G. Breit, The magnetic moment of the electron, Nature 122 (1928) 649. 
[22] J. Schwinger, On quantum-electrodynamics and the magnetic moment of the electron, Phys. Rev. 73 (1948) 416.

[23] K. Pachucki, U. D. Jentschura, and V. A. Yerokhin, Nonrelativistic qed approach to the bound-electron g factor, Phys. Rev. Lett. 93 (2004) 150401, [hep-ph/ 04108 4]. Erratum Phys. Rev. Lett. 94, 229902 (2005).

[24] K. Pachucki, A. Czarnecki, U. D. Jentschura, and V. A. Yerokhin, Complete two-loop correction to the bound-electron g factor, Phys. Rev. A 72 (2005) 022108, [phys ics / 0506227 ].

[25] M. Baranger, H. A. Bethe, and R. P. Feynman, Relativistic correction to the Lamb shift, Phys. Rev. 92 (1953) 482.

[26] R. Karplus, A. Klein, and J. Schwinger, Electrodynamic Displacement of Atomic Energy Levels. II. Lamb Shift, Phys. Rev. 86 (1952) 288-301.

[27] R. Karplus and A. Klein, Electrodynamic Displacement of Atomic Energy Levels. I. Hyperfine Structure, Phys. Rev. 85 (1952) 972-984. 